\title{
Drug Delivery Platforms for Cardiovascular Applications based on Alginate-based Hollow Structures
}

\author{
Angela Spoiala ${ }^{1}$, Cornelia Ioana Ilie ${ }^{1}$, Laura Moise², Ioana Lavinia Ardelean', Roxana Doina \\ Trusca $^{1}$, Bogdan Stefan Vasile ${ }^{1}$, Denisa Ficai ${ }^{1}$, Manuela Calin ${ }^{2}$, Anca Gafencu ${ }^{2}$, Anton Ficai ${ }^{1,3}$, Ecaterina \\ Andronescu ${ }^{1,3}$ \\ ${ }^{1}$ University POLITEHNICA of Bucharest, Faculty of Applied Chemistry and Material Science \\ 1-7 Polizu St., Bucharest, Romania \\ anton_ficai81@yahoo.com \\ ${ }^{2}$ Institute of Cellular Biology and Pathology "N. Simionescu" of Romanian Academy \\ 8, BP Hasdeu Street, 050568, Bucharest, Romania \\ ${ }^{3}$ Academy of Romanian Science \\ Splaiul Independentei Street, No 54, Bucharest, Romania
}

\section{Extended Abstract}

Over the past decade, electrospinning, a broadly used technology for electrostatic fibre formation which utilizes electrical forces to produce polymer fibres with diameters ranging from several nanometres to several micrometres using natural/synthetic polymer solutions has seen a tremendous increase in both research and commercial applications. This process offers unique capabilities for producing novel natural nanofibers and fabrics with controllable pore structure using different polymers [1-5].

Co-axial processing of alginate gels offers an alternative procedure to synthesized hollow fibres and nanobeads. The challenge of the procedure is to adequately choose the needles diameter, the flows of through the two needles, the concentrations of the solutions, etc.

Alginate/natural agent hollow fibres were successfully synthesized by coaxial electrospinning method using $3 \%$ alginate solution, $1-5 \%$ natural agent and $\mathrm{CaCl}_{2}$ solutions $(1-100 \mathrm{~g} / \mathrm{L})$ as reticulation agent. The rapid consolidation of the alginate hollow tubes is assured by passing a $\mathrm{CaCl}_{2}$ solution through the inner needle while these alginate structures are directly poured into the $\mathrm{CaCl}_{2}$ solution when hollow fibres with controlled characteristics were obtained. Natural agents such as pure polyphenols, antibiotics, analgesic, were loaded inside the wall of the alginate hallow fibres. The obtained hollow fibres were characterized by FTIR, scanning electron microscopy (SEM), tubes shrinking and water uptake. These structures will be evaluated from the point of view of loading and delivery of biological active agents such as natural or synthetic agents: antioxidants, anti-inflammatory or antimicrobial agents.

The as obtained hollow fibres were also evaluated from the point of view of biocompatibility against endothelial cells but also according to their potential antimicrobial, anti-inflammatory activity.

Keywords: Electrospinning method, Coaxial electrospinning, Alginate solution, $\mathrm{CaCl}_{2}$.

\section{Acknowledgement}

The financial contribution received from the national project "Intelligent therapies for non-communicable diseases based on controlled release of pharmacological compounds from encapsulated engineered cells and targeted bionanoparticles", PNIII-P1-1.2-PCCDI-2017-0697, Ctr. No. 13PCCDI/ 2018 is highly acknowledged.

\section{References}

[1] Nandana Bhardwaj, Subhas C. Kundu, "Electrospinning: A fascinating fiber fabrication technique," Biotechnology.

[2] Y. C. Ahn, S. K. Park, G. T. Kim, Y. J. Hwang, C. G. Lee, H. S. Shin, J. K Lee, "Development of high efficiency nanofilters made of nanofibers," Curr. Appl. Phys., vol. 6, pp. 1030-5, 2006.

[3] J. He, Y. Q. Wan, J. Y. Yu, "Scaling law in electrospinning: relationship between electric current and solution flow rate," Polymer, vol. 46, pp. 2799-801, 2005. 
[4] M. T. Hunley, "Long TE. Electrospinning functional nanoscale fibers: a perspective for the future," Polym. Int., vol. 57, pp. 385-9, 2008.

[5] J. Lannutti, D. Reneker, T. Ma, D. Tomasko, D. Farson, "Electrospinning for tissue engineering scaffolds, ” Mater. Sci. Eng. C, vol. 27, pp. 504-92, 2007. 